\title{
Spontaneous ventilation versus mechanical ventilation during video-assisted thoracoscopic surgery for spontaneous pneumothorax: a study protocol for multicenter randomized controlled trial
}

\author{
Fei Cui ${ }^{1 \#}$, Ke Xu ${ }^{1 \#}$, Hengrui Liang ${ }^{1 \#}$, Wenhua Liang ${ }^{1}, J_{i n g p e i ~}{ }^{1}{ }^{1}$, Wei Wang ${ }^{1}$, Hui Liu ${ }^{2}$, Jun Liu ${ }^{1}$, \\ Jianxing $\mathrm{He}^{1}$
}

${ }^{1}$ Department of Thoracic Surgery, First Affiliated Hospital of Guangzhou Medical University, National Respiratory Disease Clinical Research Center, Guangzhou 510120, China; ${ }^{2}$ Department of Anesthesia, First Affiliated Hospital of Guangzhou Medical University, Guangzhou 510120, China

Contributions: (I) Conception and design: All authors; (II) Administrative support: J Liu, J He; (III) Provision of study materials or patients: All authors; (IV) Collection and assembly of data: All authors; (V) Data analysis and interpretation: All authors; (VI) Manuscript writing: All authors; (VII) Final approval of manuscript: All authors.

\#These authors contributed equally to this work.

Correspondence to: Jun Liu, MD; Jianxing He, MD, PhD, FACS. Department of Thoracic Surgery, First Affiliated Hospital of Guangzhou Medical University, National Respiratory Disease Clinical Research Center, No. 151, Yanjiang Rd, Guangzhou 510120, China. Email: liujun9707@sina.com; drjianxing.he@gmail.com.

Background: With the evolution and adoption of video-assisted thoracoscopic surgery (VATS), options for anesthesia control have also seen major developments. Intubated anesthesia with single lung mechanical ventilation VATS (MV-VATS) is considered the standard of care in VATS. However, this type of ventilation strategy has been associated with several adverse effects, which can trigger complications and increase the overall surgical risk. In order to avoid intubated anesthesia related adverse effects, non-intubated spontaneous ventilation VATS (SV-VATS) strategies have been proposed in recent years and widely applied.

Methods: We established a two-arm parallel multicenter randomized controlled trial for comparative analysis of the outcomes of patients undergoing either SV-VATS or MV-VATS for spontaneous pneumothorax. Outcomes of interest include safety during operation, total analgesic dose, recovery time, postoperative complication rates, postoperative pain score, length of hospitalization, inflammation index, medical cost, etc. The recruitment target is 316 patients. Patients will be eligible if their chest CT is diagnosed with "localized lung bullae" and need VATS resection. Patients will be randomized into the SVVATS (test group) or MV-VATS (control group) after signing informed consent and surgical anesthesia evaluation.

Discussion: This protocol has been approved by the Research Ethics Committee of the First Affiliated Hospital of Guangzhou Medical university. Results will be presented at national and international meetings and conferences and published in peer-reviewed journals. We will also disseminate the main results to all participants in a letter. Non-intubated SV-VATS offered a more individual choice of anesthetics and surgical method for spontaneous pneumothorax patients.

Trial registration: NCT03016858; pre-results.

Keywords: Spontaneous ventilation; video-assisted thoracoscopic surgery (VATS); spontaneous pneumothorax; randomized controlled trial

Submitted Oct 21, 2019. Accepted for publication Feb 04, 2020.

doi: $10.21037 /$ jtd.2020.02.13

View this article at: http://dx.doi.org/10.21037/jtd.2020.02.13 


\section{Introduction}

\section{Significance}

An "overall minimally invasive" surgery (including anesthetic minimal invasiveness) developed from the use of minimally invasive incisions, has become a research hotspot in the field of minimally invasive thoracic surgery. The concept of fast track surgery (FTS) refers to a multidisciplinary approach aiming to enhance postoperative recovery, reduce complications, decrease hospital stay and reduce healthcare costs without compromising patients' safety. using a series of optimization measures with evidence-based medicine for perioperative management, including techniques and choice of anesthetics, minimally invasive surgical techniques and postoperative analgesia and rehabilitation (such as early postoperative feeding and exercise) (1) is a new concept for surgeons. In the practice of thoracic anesthesia, although good surgical field and intraoperative control can be provided using the doublelumen endotracheal tube, the anesthesia procedure for endotracheal tubing or muscle relaxant overdose can result in complications in multiple systems $(2,3)$. General anesthesia and tracheal intubation required in conventional thoracoscopy could hinder the fast recovery of the subjects, which is inconsistent with the concept of FTS. In order to minimize complications during the anesthesia procedure for endotracheal tubing and accelerate recovery, non-intubated anesthesia has been re-applied to various thoracoscopic operations $(4,5)$. Compared with intubated general anesthesia for thoracoscopic surgery, the nonintubated method could benefit in decreasing postoperative fasting time, postoperative antibiotic time and postoperative hospital stay, as well as the presence of complications (6).

\section{Current practice reviews}

In 2004, Pompeo et al. (7) first reported the successful application of spontaneous ventilation non-intubated anesthesia (thoracic epidural anesthesia in their cases) in video-assisted thoracoscopic surgery (VATS) wedge resection of pulmonary nodules. Spontaneous ventilation VATS (SV-VATS) techniques have unique advantages that can avoid the shortcomings of intubated anesthesia: (I) spontaneous ventilation can be maintained; (II) only simple intravenous drug use, local infiltration anesthesia, intercostal nerve block, or epidural block is needed for anesthesia and analgesia; (III) the use of artificial pneumothorax to achieve the effect of single lung ventilation which is necessary for VATS. Currently, non-intubated anesthesia under spontaneous ventilation has been widely applied in VATS. Intravenous anesthesia under spontaneous ventilation can avoid the residual effects of muscle relaxants, lower the incidences of systemic complications, and thus achieve faster recovery of respiratory muscle function (8). Since 2011, the First Affiliated Hospital of Guangzhou Medical University has taken the lead in the implementation of spontaneous ventilation anesthesia thoracoscopic surgery in China $(9,10)$. In 2014, our center reported a randomized controlled clinical trial that included patients that underwent SV-VATS. It is the largest study to report on fast track thoracoscopic surgery with spontaneous ventilation cases at home and abroad and results showed its safety, feasibility and advantages of more rapid recovery (8). After 5 years of trials and explorations, we have developed a variety of spontaneous intravenous combined anesthesia methods, allowing us to offer a more individual choice of anesthetics according to preoperative evaluation and surgical method for each patient, and have perform more than 2,000 cases of SV-VATS (11-16).

\section{Research purposes}

The main purpose of this trial is to evaluate the safety and feasibility of SV-VATS and physical index change during and after the operation. The primary outcome was the intraoperative surgery/anesthesia related accident rate comparison between SV-VATS and mechanical ventilation VATS (MV-VATS), which was assessed according to case of intraoperative hypoxia, intraoperative death, conversion to open surgery, conversion to intubation (only accessed in SV-VATS group) and other intraoperative adverse events could be happened in two groups. Other study outcomes included changes of vital sign, total analgesic dose, recovery time, post-operative complication rates, post-operative pain scores, length of hospitalization, inflammation index, costeffectiveness evaluation, etc.

\section{Study design and sample size}

Subjects are admitted to the hospital for surgical treatment, and random allocation is performed after signing informed consent and surgical anesthesia evaluation. According to the randomized results, they will be grouped into the SVVATS group (test group) or MV-VATS (control group). The schematic outline of the trial design was showed in Figure 1. 


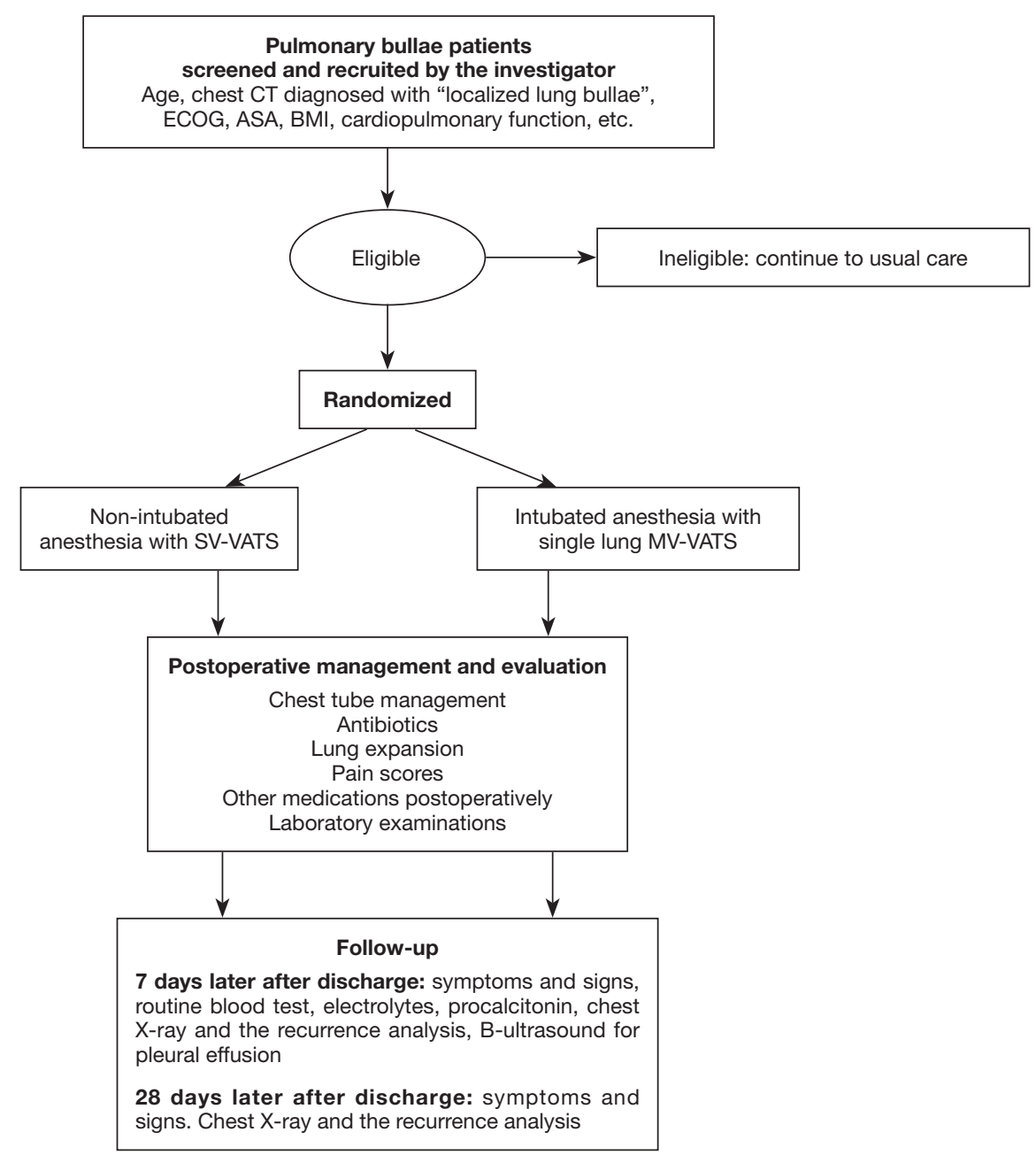

Figure 1 Schematic outline of the trial design. CT, computed tomography; ECOG, Eastern Cooperative Oncology Group score standard; ASA, American Society of Anesthesiologists score; BMI, body mass index. MV-VATS, mechanical ventilation VATS; SV-VATS, spontaneous ventilation VATS.

\section{Sample size calculation}

According to previous meta-analysis from our team (8), the postoperative complication rate of MV-VATS was $27 \%$, the non-inferiority margin was set as $15 \%$, which means the upper limit of the $95 \%$ confidence interval (CI) for the estimated difference in the postoperative complication rate between the SV-VATS group and the MV-VATS group exceeded $15 \%$, the SV-VATS would be inferior to the MVVATS group. With single-sided $\alpha$ risk of 0.05 and the dropout rate of $10.98 \%$, the sample size required to detect a difference was 316 patients in total, with each group of 158 patients in this study. All primary and secondary data were analyzed according to an intention-to-treat principle. We used Student's $t$ test for parametric means to compare means, and $\chi^{2}$ tests or Fisher exact tests to compare proportions. We did not attempt to estimate missing data. All patients, including those with missing values, were included in the analyses using the data available. The primary analysis will be by intention to treat (ITT).

\section{Participants selection}

\section{Inclusion criteria}

Patients will be recruited from the out-patient clinics at 8 centers that included the First Affiliated Hospital of Guangzhou Medical University, Nanfang Hospital of Southern Medical University, the Second Affiliated Hospital, South China University, the Third People's 
Hospital, Shenzhen, Affiliated Hospital of Southwest Medical University, Taizhou Hospital, Zhejiang Province, the First Affiliated Hospital, Sun Yat-sen University and the Jieyang People's Hospital, Guangdong Province. Patients are eligible for the study if they fit the following criteria: (I) volunteer to participate in clinical trials and have the ability to responsibility sign informed consent; (II) $16-50$ years old; (III) preoperative chest CT diagnosed with "localized lung bullae" and in need of surgical treatment; (IV) Eastern Cooperative Oncology Group (ECOG) score standard $\leq 1$; (V) American Society of Anesthesiologists (ASA) score $\leq 2$; (VI) cardiopulmonary function and other important organ functions are basically normal and can endure surgery; (VII) if antibiotics have been used pre-surgery, at least a 24-hour interval from stopping to the preoperative prevention use of antibiotics infusion should be maintained. If analgesic medication has been used preoperatively, at least a 24-hour interval from stopping to the start of surgery should be maintained.

\section{Exclusion criteria}

Exclusion criteria is as follows: (I) refuses to participate in clinical trials; (II) has a history of disease or surgery or symptoms of increased postoperative drainage, such as a history of tuberculosis, a history of ipsilateral thoracic surgery, moderate or high level pleural effusion indicated by B-mode ultrasound preoperatively; or pulmonary infection etc.; or other history or manifestations that could cause postoperative air leaks, such as diffuse emphysema; (III) body mass index (BMI) $\geq 25$; (IV) other situations such as absolute surgical contraindications, menstrual period, pregnancy or other conditions considered not suitable for recruitment by investigators; (V) allergy to penicillin, cephalosporin, and morphine or any drugs involved in programs.

\section{Baseline data collection}

For recruited patients, baseline data will be collected including gender, age, history of respiratory system and other systemic diseases, as well as main results of physical, laboratory and instrumental examinations such as: preoperative blood test: routine blood test, blood type, procalcitonin, liver function (ALT, AST, GGT, TBIL, DBIL, albumin), renal function (BUN or urea, Cr), electrolytes $\left(\mathrm{Na}^{+}, \mathrm{Cl}^{-}, \mathrm{Ca}^{2+}, \mathrm{K}^{+}\right)$, coagulation function (APTT, PT, PT-INR), arterial blood gas analysis $\left(\mathrm{PO}_{2}\right)$
$\mathrm{PCO}_{2}, \mathrm{PH}$ ), blood transmission (hepatitis $\mathrm{B}$ surface antigen, hepatitis C antibody, syphilis antibody, HIV antibody), female urine or blood HCG. Preoperative examination: ECG, echocardiography, chest X-ray, chest CT scan.

\section{Randomization}

We use the SAS V.9.2 software package (SAS Institute, Cary, North Carolina, USA) to generate random numbers in a $1: 1$ ratio, with a block size of 4 . Randomization is stratified by centers. The results are sealed in envelops and stored at the study site until the end.

A study coordinator assigned from each center saves and distributes randomization results according to the order of recruited patients, and coordinates among the investigators. Each enrolled patient will be grouped in the operating room on the day of surgery, the designed anesthesiologist will perform anesthesia management and intraoperative data collection. Postoperative follow-up will be performed by researchers who have not been involved in patient care and have received follow-up training. Both anesthesiologists and researchers do not communicate with each other when collecting data.

\section{Interventions}

\section{Preparation of anesthesia}

Before the anesthesia, patients cannot eat for $8 \mathrm{hrs}$ or drink for 6hours prior to surgery preparation. Patients electrocardiogram (ECG), heart rate (HR), non-invasive blood pressure (BP), pulse oximetry $\left(\mathrm{SpO}_{2}\right)$, respiratory rate (RR), bispectral index (BIS), and end tidal carbon dioxide $\left(\mathrm{PetCO}_{2}\right)$, inhaled oxygen fraction $\left(\mathrm{FiO}_{2}\right)$, and neuromuscular transmission (used in the conventional intubated anesthesia group) are continuously monitored in the operating room.

The third-generation double-tube laryngeal mask is used in the SV-VATS group for 3\# (30-50 kg), 4\# (50-70 kg) or $5 \#(70-100 \mathrm{~kg})$ while the double lumen bronchial catheter (Mallinckrodt) is used in the MV-VATS group, the diameter is selected according to the tracheal diameter at the sternoclavicular joint level from the chest X-ray.

Subjects will receive a continuous intravenous infusion of 1 dose of cefuroxime 30-60 minutes before surgery to prevent postoperative infections. The BIS sensor is used for evaluation of sedation level and advanced judgement of the sedation depth for both groups to 
Table 1 Anesthesia effect score

\begin{tabular}{ll}
\hline Scores & Anesthesia effects \\
\hline 4 & Complete, painless and quiet anesthesia, providing good conditions for surgery, and hemodynamics remains relatively stable \\
3 & $\begin{array}{l}\text { Slightly incomplete anesthesia with mild pain, not very good muscle relaxation, sedative is needed, and fluctuating } \\
\text { hemodynamics (not caused by the disease) are observed }\end{array}$ \\
2 & $\begin{array}{l}\text { Incomplete anesthesia with obvious pain, moan and inciting, and the situation is improved after the medication, } \\
\text { but it is not ideal, and barely for operation performance }\end{array}$ \\
& Anesthesia conversion is required for surgery performance \\
\hline
\end{tabular}

Table 2 Consciousness level

\begin{tabular}{ll}
\hline Degree & Consciousness level \\
\hline 0 & The patient is asleep and no response to calling \\
1 & The patient is asleep, body movements or blinking, head and neck movements are presented when calling \\
2 & The patient is awake and has a level 1 performance, can open mouth and stick out tongue \\
3 & The patient is awake and has a level 2 performance and can tell his/her age or name \\
4 & The patient is awake and has a level 3 performance and can recognize the people around or know where he/she is \\
\hline
\end{tabular}

monitor the level of consciousness. Neuromuscular transmission is only monitored in the MV-VATS group. TOF-WACTH or TOF-GUARD, the internationally recognized neuromuscular transmission instruments, are used. Monitoring the $\mathrm{TOF}_{\mathrm{r}}$ value can standardize the intraoperative dose and time of the muscle relaxant, so as to obtain the tracheal tube extubation time, postoperative muscle strength (grip strength test) and the time of ambulation under the same conditions.

\section{Anesthesia regimen and process}

We use the scores listed in Table 1 to assess the effects of anesthesia and use the criteria listed in Table 2 to assess the consciousness level of patients.

\section{Anesthesia regimen of SV-VATS group Anesthesia induction}

For anesthesia induction, after dexmedetomidine is pumped at a rate of $1.0 \mu \mathrm{g} / \mathrm{kg} / \mathrm{h}$ for 15 minutes, infusion of propofol (TCI) 2- and sufentanil are started at $3.5 \mu \mathrm{g} / \mathrm{mL}$ and $0.2 \mu \mathrm{g} / \mathrm{kg}$ respectively. When the BIS value drops to 60 or less, to observe the respiratory condition, we place the laryngeal mask and connect to the breathing circuit. If there is no spontaneous ventilation, manual ventilation or simultaneous intermittent mandatory ventilation (SIMV) mode is used to assist ventilation $\left(\mathrm{FiO}_{2} 1, \mathrm{~V}_{\mathrm{T}} 7-8 \mathrm{~mL} / \mathrm{kg}\right.$, RR 10-12 breaths/min, oxygen flow 4-5 L/min), and $\mathrm{PetCO}_{2}$ is monitored. Non-surgical radial artery puncture is performed to continuously monitor invasive arterial blood pressure (no central venipuncture required).

\section{Anesthesia maintenance and intraoperative airway management}

For anesthesia maintenance period, before incision of the skin, propofol (TCI) is administered at $1.5-4 \mu \mathrm{g} / \mathrm{mL}$, remifentanil at $0.03-0.08 \mu \mathrm{g} / \mathrm{kg} / \mathrm{min}$, and dexmedetomidine at $0.5 \mu \mathrm{g} / \mathrm{kg} / \mathrm{h}$ respectively to maintain the BIS value between 45 and 60 . If the invasive blood pressure SBP $<90 \mathrm{mmHg}$, intravenous dopamine can be injected, the initial amount is $3-5 \mu \mathrm{g} / \mathrm{kg} / \mathrm{min}$, and the maximum amount is $<8 \mu \mathrm{g} / \mathrm{kg} / \mathrm{min}$. Dexmedetomidine is stopped directly after the pleural cavity closure, and propofol and remifentanil are stopped at the end of the operation. The anesthetic is not inhaled throughout the procedure.

\section{Intraoperative airway secretion management}

Suction of sputum through the laryngeal mask under spontaneous ventilation should maintain negative pressure $<10 \mathrm{kPa}$, and $<10 \mathrm{~s}$ at each time, do not repeatedly stimulate the glottis to prevent cough and laryngeal spasm.

\section{Anesthesia techniques}

The anesthesia techniques consist of local anesthesia, surface anesthesia and nerve block anesthesia. The local 
anesthesia positions are at the upper edge and the lower edge of the intercostal space where the incision is located. The peripheral anesthesia range of the incision is $2 \mathrm{~cm}$, and the anesthesia is infiltrated with $5 \mathrm{~mL} 1 \%$ lidocaine.

Surface anesthesia is given by $5 \mathrm{~mL} 2 \%$ lidocaine that is sprayed on the visceral pleural surface. Nerve block anesthesia is performed by $2.5 \mathrm{~mL} 0.5 \%$ ropivacaine and $2.5 \mathrm{~mL} 2 \%$ lidocaine and are infused to block the vagus nerve. If anatomical abnormalities are found during surgery that may cause difficulty performing the vagus nerve block, such as a right aortic arch, an ectopic venous arch, etc., the vagus block anesthesia cannot be performed. After the anesthesia is completed, remifentanil can be reduced to $0.03-0.05 \mu \mathrm{g} / \mathrm{kg} / \mathrm{min}$.

\section{Air leakage test}

At the end of surgery, the operated lung was manually ventilated through the laryngeal mask to check for air leakage. Some difficulty expanding the lung using the laryngeal mask during air leakage test resulting in atelectasis is possible but can be reduced by pressing both sides of the larynx lightly.

\section{Intraoperative treatment in special cases}

Mediastinal flutter after artificial pneumothorax. If the amplitude of mediastinum flutter is obvious, the operation will be affected. The main reason is the effect of singlepulmonary respiration and anesthesia drugs (especially Remifentanil and Propofol) on respiration after artificial pneumothorax. The ideal parameter of single-lung spontaneous respiration is $\mathrm{V}_{\mathrm{T}} 3-4 \mathrm{~mL} / \mathrm{kg}$, RR of $20-25$ per minute. Because Dexmedetomidine has relatively less effect on respiration, Dexmedetomidine is kept at a constant rate intraoperatively. The tidal volume and respiratory rate of spontaneous respiration will be altered by Propofol rate adjustment. While the BIS value is maintained at 45-60. The improvement of local anesthesia + nerve block anesthesia has contributed to the gradual reduction of the dose of Remifentanil, to downgrade the possibility of interference with breathing.

Intraoperative hypercapnia. Hypercapnia is common during surgery, but it is well tolerated. When $\mathrm{PaCO}_{2}$ $\geq 80 \mathrm{mmHg}$, manual or intermittent SIMV mode ventilation $\left(\mathrm{FiO}_{2} 1, \mathrm{~V}_{\mathrm{T}} 3-5 \mathrm{~mL} / \mathrm{kg}\right.$, RR 12-15 times/min, oxygen flow 4-5 L/min) can be used to reduce the hypercapnia, while adjusting the speed of Propofol and Remifentanil. If the above treatment fails to improve hypercapnia, conversion of anesthesia is considered.

Intraoperative hypoxemia. The incidence of hypoxemia is low. It usually occurs after the complete collapse of the lung on surgical side. If $\mathrm{SpO}_{2}<90 \%$, manual or intermittent SIMV mode ventilation $\left(\mathrm{FiO}_{2} 1, \mathrm{~V}_{\mathrm{T}} 3-5 \mathrm{~mL} / \mathrm{kg}, \mathrm{RR}\right.$ 12-15 times/min, oxygen flow $4-5 \mathrm{~L} / \mathrm{min}$ ) can be used. When the lung on the surgical side is completely collapsed, the airway resistance of the operation side is higher than that of the contralateral side, and most of the gas will enter the contralateral lung when ventilated by small tidal volume. Generally, small tidal volume ventilation does not cause lung expansion on the surgical side and has little effect on the surgical operation.

Laryngeal mask shift. If inspiratory dyspnea occurs during surgery, the $\mathrm{PetCO}_{2}$ waveform drops flat or disappears, and $\mathrm{V}_{\mathrm{T}}$ drops suddenly, the possibility of a laryngeal mask shift is considered. In this case it is necessary to deepen anesthesia appropriately and adjust the position of the laryngeal mask to relieve the obstruction.

\section{Induction of spontaneous respiratory recovery}

Spontaneous respiratory recovery can be induced by manual controlled breathing. If spontaneous ventilation cannot be recovered within $10 \mathrm{~min}$, the dose of the anesthetic/ analgesic can be reduced. Until spontaneous ventilation is restored, the oxygen concentration is then adjusted to $100 \%$ and the oxygen flow rate is adjusted to $4-5 \mathrm{~L} / \mathrm{min}$.

\section{Timing of laryngeal mask removal}

When the patients' conditions meet the following criteria, the laryngeal mask should be removed:

(I) Being conscious, the patients can blink when called;

(II) Air inhalation with a laryngeal mask: $\mathrm{SpO}_{2}>95$ lasting about 5 to 10 minutes, (or $\mathrm{PaO}_{2}>85 \mathrm{mmHg}$, $\mathrm{PaCO}_{2}<50 \mathrm{mmHg}$;

(III) $\mathrm{V}_{\mathrm{T}}>6-8 \mathrm{~mL} / \mathrm{kg}$;

(IV) Stable circulation.

\section{Anesthesia regimen of the MV-VATS group Anesthesia induction}

After dexmedetomidine is pumped at a rate of $1.0 \mu \mathrm{g} / \mathrm{kg} / \mathrm{h}$ for 15 minutes, $2-3.5 \mu \mathrm{g} / \mathrm{mL}$ Propofol (TCI) is given via target-controlled infusion (TCI) in combination with $0.3 \mu \mathrm{g} / \mathrm{kg}$ intravenous infusion Sufentanil and $0.2 \mathrm{mg} / \mathrm{kg}$ intravenous infusion cis-atracurium. After 180 seconds, double lumen bronchial intubation is performed, fiberoptic bronchoscopy is used for positioning. While arterial catheterization is performed at the radical artery on the non-operative side to continuously monitor invasive arterial blood pressure (no central venipuncture required).

\section{Anesthesia maintenance}

Before any operative incision of the skin, continuous intravenous infusion of $1.5-4 \mu \mathrm{g} / \mathrm{mL}$ propofol is 
performed via TCI Continuous infusion of remifentanil 0.03-0.08 $\mu \mathrm{g} /(\mathrm{kg} \cdot \mathrm{min})$, Dexmedetomidine hydrochloride $0.5-1.0 \mu \mathrm{g} /(\mathrm{kg} \cdot \mathrm{h})$ and Cis-atracurium $0.05 \mathrm{mg} / \mathrm{kg}$ $(1 *$ ED95) is administered to maintain the BIS value between 45-60. If the invasive blood pressure SBP $<90 \mathrm{mmHg}$, intravenous dopamine can be injected, the initial amount is $3-5 \mu \mathrm{g} / \mathrm{kg} / \mathrm{min}$, and the maximum amount is $<8 \mu \mathrm{g} /(\mathrm{kg} \cdot \mathrm{min})$. Dexmedetomidine is stopped immediately after the pleural cavity is closed, and propofol and remifentanil are stopped at the end of the operation. The anesthetic is not inhaled throughout the procedure. Parameters during single lung ventilation should be under intermittent positive pressure ventilation (IPPV) mode as $\mathrm{FiO}_{2} 1, \mathrm{~V}_{\mathrm{T}}$ 6-8 mL/kg, RR 12-18 times/min, oxygen flow 4-5 L/min.

\section{Anesthesia recovery and extubation}

When spontaneous respiration is resumed, intravenous injection of $0.02 \mathrm{mg} / \mathrm{kg}$ neostigmine and $0.01 \mathrm{mg} / \mathrm{kg}$ atropine to antagonize the muscle relaxant's effect. If patients' heart rate $\geq 100$ beats $/ \mathrm{min}$, neostigmine alone is chosen as antagonists without atropine.

\section{Extubation indications are recommended as following:}

(I) $\quad \mathrm{TOF}_{\mathrm{r}}>0.9$;

(II) Being conscious, the patients can blink when called;

(III) Air inhalation with bronchial tube: $\mathrm{SpO}_{2}>95$ for 5-10 minutes (or $\mathrm{PaO}_{2}>85 \mathrm{mmHg}, \mathrm{PaCO}_{2}$ $<50 \mathrm{mmHg}$ );

(IV) $\mathrm{V}_{\mathrm{T}}>6-8 \mathrm{~mL} / \mathrm{kg}$;

(V) Stable circulation.

\section{Anesthesia conversion}

If hypoxemia or hypercapnia or other indicated conditions for conversion occurs during the surgery and cannot be resolved after non-invasive management, the anesthesiologist must be able to switch the anesthesia mode and perform tracheal intubation. The single lumen endotracheal tube + bronchial blocker is preferred. Given there is Intra-airway hemorrhage, lung isolation can be achieved by double lumen endotracheal intubation.

The single-lumen tube should be inserted under the guidance of a fiber optic bronchoscope when the patient is in a lateral position, which is more difficult than normal practice. To achieve this a small pillow should be placed under the head to allow the front, bottom perspective of the mouth and nose to stay up and the head and neck should be parallel to the central axis of the body.

Indications for anesthesia conversion

(I) Hypoxemia: $\mathrm{SpO}_{2}<90 \%$, no improvement of blood oxygen saturation after auxiliary ventilation;

(II) $\mathrm{PaCO}_{2} \geq 80 \mathrm{mmHg}$, no improvement of hypercapnia after auxiliary ventilation, and any of the following:

* Circulation change: HR >100 bpm, or the change of systolic pressure amplitude $>30 \%$ of the base value;

* Arrhythmia occurs, such as frequent atrial or ventricular premature $\geq 6$ beats/min (excluding from surgical stimulation induced arrhythmia);

* Arterial blood gases analyses are detected twice at intervals of $15 \mathrm{~min}$ or more, and the $\mathrm{pH}$ values are all $<7.25$.

(III) The swing amplitude of surgical field is large which is difficult to perform surgical operation, and is not improved after drug treatment, duration $>5 \mathrm{~min}$;

(IV) Severe hemorrhage in the surgical wound and thoracic cavity blurred surgical field;

(V) A significant increase in endotracheal secretions, especially bloody secretions which cause difficulty in breathing, increased airway resistance, the reduction of spontaneous ventilation $\mathrm{V}_{\mathrm{T}}>30 \%$, mechanical ventilation $\mathrm{P}_{\text {peak }}>20 \mathrm{cmH}_{2} \mathrm{O}$;

(VI) After surface anesthesia and local anesthetic block of the intrathoracic vagus nerve is achieved, coughing still occurs $>2$ times/min;

(VII) Those who meet the exclusion criteria of this study may consider anesthesia conversion.

\section{Surgical procedure}

After artificial pneumothorax, the collapse of the operative lung, required for the optimal visualization of the surgical field during VATS. We use the grades listed in Table 3 to assess the surgical exposure.

\section{SV-VATS group}

Local injection is performed with $5 \mathrm{~mL} 1 \%$ Lidocaine before the incision of skin. The approach for the VATS resection of bullae is the single port method, an incision $\leq 2 \mathrm{~cm}$ is made in the $4^{\text {th }}$ or $5^{\text {th }}$ intercostal space on the anterior axillary line as the working port. Intrathoracic spray of $5 \mathrm{~mL} 2 \%$ Lidocaine is used, and $2.5 \mathrm{~mL} 0.5 \%$ Ropivacaine $+2.5 \mathrm{~mL} 2 \%$ Lidocaine is used to block the thoracic vagus nerve. No pleurodesis is needed intraoperatively. A $2 \mathrm{kPa}$ pressure is applied in conjunction with laryngeal mask ventilation to expand the lung in order to observe if there is any leakage. A 20-F chest tube is placed on the top of chest. Patients are restored to a horizontal position after 
Table 3 The grades of surgical exposure

\begin{tabular}{ll}
\hline Grades & Surgical field exposure and lung collapse \\
\hline 5 & A clear surgical field exposure, a satisfactory lung collapse \\
4 & A less clearly surgical field exposure, a fine lung collapse, no intermittent surgery is needed \\
3 & $\begin{array}{l}\text { A fine surgical field exposure, an unsatisfactory lung collapse, intermittent surgery to adjust anesthesia indicators such as tidal } \\
\text { volume is needed }\end{array}$ \\
2 & A poor surgical field exposure, an unsatisfactory lung collapse, intermittent surgery is needed in most cases \\
1 & A very poor surgical field exposure that is unable to perform surgery, conversion to endotracheal intubation is needed
\end{tabular}

the pleural cavity is closed, then $2 \mathrm{kPa}$ negative pressure is used for air exhausting until no obvious bubble overflow is found.

\section{MV-VATS group}

A single port is used as the approach for VATS resection of bullae, the working port is made in the $4^{\text {th }}$ or $5^{\text {th }}$ intercostal space at the anterior axillary line. No pleurodesis is needed intraoperatively. Two $\mathrm{kPa}$ pressure is applied in conjunction with laryngeal mask ventilation to expand the lungs in order to observe if there is any leakage. A 20-F chest tube is placed on the top of chest. The patients are restored to a horizontal position after the pleural cavity is closed, then $2 \mathrm{kPa}$ negative pressure is used for air exhausting until no obvious bubble overflow is found.

\section{Monitoring}

During both anesthesia induction, and the operation, continuous monitoring of blood gas analysis, ECG, HR, IBp, $\mathrm{SpO}_{2}, \mathrm{RR}, \mathrm{BIS}, \mathrm{PetCO}_{2}, \mathrm{FiO}_{2}$ and muscle relaxation monitoring (used in the MV-VATS group) is standard, and is recorded after preoperative intravenous anesthesia (after intubation for MV-VATS group), after the pleural cavity is opened, 15 minutes after opening the pleural cavity, 30 minutes after opening the pleural cavity, every hour after the pleural cavity is opened, after the pleural cavity is closed, and after complete recovery to consciousness.

During the operation, lung manipulation can trigger a cough reflex, so the proportion of stretching of pulmonary lobes and induced coughing intraoperatively should be recorded if it occurs.

The time for post-anesthesia care unit (PACU) refers to the time from which the anesthesia is stopped to the Steward score $\geq 4$ which considers awakening, airway patency and limb activity. A Steward score $\geq 4$ is considered as fully awake. The criteria of Steward score were showed in Table 4.

\section{Postoperative management and evaluation}

\section{Postoperative analgesia}

Patient controlled analgesia (PCA) is used after the operation, morphine is diluted with $0.9 \%$ sodium chloride to $100 \mathrm{~mL}$, the morphine concentration is $1 \mathrm{mg} / \mathrm{mL}$. the loading dose is $0 \mathrm{~mL}$, backgrounding infusion dose is $0 \mathrm{~mL}$, the bolus is $1 \mathrm{~mL}$, the lockout time is $10 \mathrm{~min}$, and pressing time $\leq 6 /$ hour.

We use the Visual Analogue Scale (VAS) to analyze the analgesic efficacy and side effects of the operation.

VAS (0-10 points): 0 points: no pain; 3 points or less: mild pain, and it is endurable; $4-6$ points: moderate pain, affecting sleep, could be tolerable; $7-10$ points: the patients have progressively strong pain and the pain is unbearable, affecting appetite and sleep.

Investigators should consider the degree of pain when selecting analgesics based on the principle: non-steroidal anti-inflammatory drugs (preferred) $\rightarrow$ central analgesics (secondary preferred) $\rightarrow$ anesthetic analgesics. It is recommended that the VAS pain score is $\geq 4$ points under the condition that an analgesic pump has been used before the use of analgesic drugs is considered and recorded as an AE (Adverse Event).

\section{Chest tube management}

The chest tube is extubated after reexamining the chest $\mathrm{X}$-ray within 4 hours postoperatively (chest X-ray examination and chest tube extubation: window period \pm 2 hours) if there is no obvious air leakage and no active bleeding, the chest $\mathrm{X}$-ray suggests good lung recruitment.

But thoracentesis after extubating is required due to poor 
Table 4 Steward score

\begin{tabular}{l}
\hline Criteria of Steward score \\
\hline Level of awake \\
Fully awake \\
Response to stimulation \\
No response to stimulation \\
Degree of airway patency \\
Can be coughed according to the doctor's instructions \\
Self-airway patency maintenance without auxiliary support \\
Auxiliary support is required for airway patency maintenance \\
Degree of limb activity \\
Consciously limb activity \\
Unconscious limb activity \\
No limb activity
\end{tabular}

lung expansion or pleural effusion postoperatively if the degree of lung expansion is intermediate and below, or the amount of pleural effusion reaches the middle and above.

\section{Management of antibiotics}

If antibiotics had been used before surgery, the interval between the deactivation times to the start of infusion of preoperative prophylaxis should be at least 24 hours. Only one dose of antibiotics was given before the start of surgery, and no antibacterial drug is used after surgery; if the operation time exceeds 3 hours, or the blood loss is larger than 1,500 $\mathrm{min}$ ), the second dose of antibiotics should be given during surgery.

If meeting three of the following five items, after comprehensive evaluation and discussion with the project team, antibiotic therapies can be used postoperatively:

(I) Chest $\mathrm{X}$-ray showed significant infiltration or exudation;

(II) Temperature is above $38.5^{\circ} \mathrm{C}$;

(III) Airway purulent secretions;

(IV) Postoperative leukocytes greater than $11 \times 10^{9} / \mathrm{L}$ or more;

(V) Procalcitonin is elevated, greater than $2 \mathrm{ng} / \mathrm{mL}$ within 4 hours after surgery, or greater than $0.5 \mathrm{ng} / \mathrm{mL}$ after 24 hours after surgery.

If meeting any item of the following criteria, use of antibiotics should be discontinued:
(I) Cheat X-ray showed pulmonary exudation is improved;

(II) The temperature below $38.5^{\circ} \mathrm{C}$;

(III) Absence of airway purulent secretions;

(IV) Postoperative Leukocytes below $11 \times 10^{9} / \mathrm{L}$;

(V) Procalcitonin concentration declines to $0.5 \mathrm{ng} / \mathrm{mL}$ or less.

\section{The comparison of postoperative lung expansion}

Chest X-ray is recommended as an evaluated method and should be performed during hospitalization (within 4 hours after surgery, within 48 hours after surgery, and every 72 hours after surgery, 1 week after discharge and 4 weeks after discharge respectively. According to the degree of postoperative lung recruitment, the lung expansion degree is divided into "good" (lung expansion $\geq 70 \%$ ), "medium" ( $70 \%>$ lung expansion $\geq 50 \%$ ), and "poor" (lung expansion $<50 \%)$.

\section{Grip strength test}

Grip strength test is used to evaluate muscle weakness and should be monitored as following: before the operation, 2, 4 and 6 hours after surgery. Each patients' left and right grip strength is tested three times respectively, then the average value is taken (keep 1 decimal place, round off), $\mathrm{kg}$ is used as units uniformly. 


\section{Discharge}

Discharge of any subjects is not allowed until reaching the following criteria:

(I) The submaxillary temperature below $38^{\circ} \mathrm{C}$;

(II) Blood oxygen saturation $>90 \%$ without oxygen inhalation;

(III) No respiratory infections and no complications requiring hospitalization;

(IV) After extubating, thoracic radiology showed more than $70 \%$ of lungs are re-expanded or pleural effusion is below the medium amount (Standard as described above).

The postoperative hospital stay should be recorded accordingly.

\section{Follow-up}

Recorded indicators of outpatient examination at 7 days after discharge (window period \pm 3 days) include: (I) symptoms and signs; (II) routine blood test, electrolytes, procalcitonin, chest $\mathrm{X}$-ray and the recurrence analysis, B-ultrasound for pleural effusion if necessary (decided by the investigator). At 28 days after discharge (window period \pm 3 days) examination includes: (I) symptoms and signs; (II) chest $\mathrm{X}$-ray and the recurrence analysis

If the subject is re-admitted within 1 week after discharge for the following reasons, it must to be recorded.

(I) Fever $\left(>38.5^{\circ} \mathrm{C}\right)$, the oxygen saturation is less than $90 \%$ without oxygen inhalation;

(II) Leukocytes greater than $15 \times 10^{9} / \mathrm{L}$ or more;

(III) The lung expansion is less than $70 \%$, and the amount of pleural effusion is medium and above (standard as described above);

(IV) Re-admission for further treatment agreed by other investigators.

\section{Data management}

This study used the paper case report form (CRF) for the data transfer and delivery. After the study begins, CRF revision should be performed if there is any missing necessary data items or ambiguous items in CRF, without medical and economic risk and burden increasing, after discussion of the research committee, whereas the CRF revision without the study treatment protocol revision should not revise the research protocol. Submission or re-application of revised CRF files to the IRB of the participating hospitals should be carried out in accordance with the regulations of each hospital.

Protocol violation (PV) is defined as any changes and non-compliance with the design or process of the clinical trial protocol under the management of the investigator, or any actions made without approval of the Institutional Review Board (IRB), and can be divided into: "general protocol violation (minor PV)" and "critical protocol violation (major PV)" according to its' severity.

Common general PVs include: (I) events presented outside the time window of visits/observations/examinations but did not affect to continue use of the study drug as planned, or the effectiveness of evaluation of primary efficacy point and key secondary efficacy point; (II) missing data resulting from loss of the data points or laboratory parameters according to the protocol but does not affect outcomes of primary efficacy point or critical secondary efficacy or safety points. For example, the specified indicators in the protocol are not listed in the CRF, or a research institution does not have the inspection conditions of a laboratory indicator; (III) incomplete observation/ evaluation but does not affect primary or key secondary efficacy or safety outcomes, such as missing blood pressure measurements.

When a PV is found, it should be recorded using the "Protocol Violation Record Form", and recorded in detail including the discovery time, the duration and process of the event, the reason and the corresponding treatment measures, and notification of the ethics committee and the Study Sponsor. After confirmation by the investigator, the event form should be sent by e-mail to the sponsor to determine whether the subject can continue to conduct the test and evaluate the degree of violation. The general violation report ethics committee can report according to the time limit of the local ethics committee or monthly, and report immediately when the presence of critical PV.

\section{Adverse events}

Any adverse medical event that occurred between the times the subject signed the informed consent of the test and the last follow-up is determined to be an adverse event regardless of whether the event and the test had a causal relationship (except laboratory abnormal values, laboratory abnormal AE are defined as follows). The investigator should follow up on all adverse events until the symptoms disappear or the condition is stable. Adverse events are recorded during the trial, including the time, severity, 
ending time, measures taken, and progress and outcomes of the adverse events.

\section{The laboratory abnormal AE definition}

If the laboratory outliers such as blood pressure, blood glucose, blood lipid levels and so on occurred after the signing of the informed consent form and before randomization: abnormally and clinically meaningful values are classified as medical history, those without clinical significance did not belong to the $\mathrm{AE}$ and medical history. If laboratory outliers such as routine blood test, electrolytes, procalcitonin, blood oxygen saturation and so on occurred after randomization: abnormal and clinically meaningful values are $\mathrm{AE}$, those that are not clinically significant are not $\mathrm{AE}$.

\section{Evaluation criteria for severity of adverse events (17)}

(I) Mild: does not affect the daily activities of the subject;

(II) Moderate: to some extent affect the daily activities of the subject;

(III) Severe: significantly affects the daily activities of the subject.

\section{Severe adverse events}

The occurrence of any of the following conditions should be considered as a severe adverse event: (I) hospitalization for further treatments; (II) extends the length of hospital stay; (III) disability; (IV) affects work ability; (V) lifethreatening or death; (VI) causes congenital malformations;

In the presence of severe adverse events, the investigator should immediately take appropriate treatment measures to protect the safety of the subject. The research leader should be notified immediately within 24 hours. The research leader should actively handle the severe events, fill out the "SAE Form", and report to the State Food and Drug Administration, the Provincial Food and Drug Administration, the National Health and Family Planning Commission, the Ethics Committee of the Hospital, and the clinical trial institutions and sponsors of the hospital within 24 hours. Finally, the ethics committee decides whether to suspend the trial or not.

\section{Cost-effectiveness evaluation}

The cost-effectiveness evaluation was based on data from all patients who enrolled and completed the study. The mean total cost was evaluated in the SV-VATS and MV-VATS group. The specific costs of anesthesia, operation, nursing, medicine, surgical consumables, diagnostic, physiotherapy and other costs were also evaluated.

\section{Study dissemination}

The results will be presented at national and international meetings and conferences and published in peer-reviewed journals. We will also disseminate the main results to all participants in a letter.

\section{Acknowledgments}

Funding: This work was supported by Guangzhou Institute of Respiratory Disease.

\section{Footnote}

Conflicts of Interest: All authors have completed the ICMJE uniform disclosure form (available at http://dx.doi. org/10.21037/jtd.2020.02.13). JH serves as an unpaid Editor-in-Chief of Fournal of Thoracic Disease. WL serves as an unpaid editorial board member of Fournal of Thoracic Disease from Dec 2015 to Dec 2021. The other authors have no conflicts of interest to declare.

Ethical Statement: The authors are accountable for all aspects of the work in ensuring that questions related to the accuracy or integrity of any part of the work are appropriately investigated and resolved. The study was approved by the Research Ethics Committee of the First Affiliated Hospital of Guangzhou Medical University (SVA-001) and written informed consent was obtained from all patients.

Open Access Statement: This is an Open Access article distributed in accordance with the Creative Commons Attribution-NonCommercial-NoDerivs 4.0 International License (CC BY-NC-ND 4.0), which permits the noncommercial replication and distribution of the article with the strict proviso that no changes or edits are made and the original work is properly cited (including links to both the formal publication through the relevant DOI and the license). See: https://creativecommons.org/licenses/by-nc-nd/4.0/.

\section{References}

1. Kehlet H, Wilmore DW. Evidence-based surgical 
care and the evolution of fast-track surgery. Ann Surg 2008;248:189-98.

2. Campos JH, Massa FC, Kernstine KH. The incidence of right upper-lobe collapse when comparing a rightsided double-lumen tube versus a modified left doublelumen tube for left-sided thoracic surgery. Anesth Analg 2000;90:535-40.

3. Kim JH, Park SH, Han SH, et al. The distance between the carina and the distal margin of the right upper lobe orifice measured by computerised tomography as a guide to right-sided double-lumen endobronchial tube use. Anaesthesia 2013;68:700-5.

4. Chen JS, Cheng YJ, Hung MH, et al. Nonintubated thoracoscopic lobectomy for lung cancer. Ann Surg 2011;254:1038-43.

5. Tseng YD, Cheng YJ, Hung MH, et al. Nonintubated needlescopic video-assisted thoracic surgery for management of peripheral lung nodules. Ann Thorac Surg 2012;93:1049-54.

6. Liu J, Cui F, Pompeo E, et al. The impact of nonintubated versus intubated anaesthesia on early outcomes of video-assisted thoracoscopic anatomical resection in non-small-cell lung cancer: a propensity score matching analysis. Eur J Cardiothorac Surg 2016;50:920-5.

7. Pompeo E, Mineo D, Rogliani P, et al. Feasibility and results of awake thoracoscopic resection of solitary pulmonary nodules. Ann Thorac Surg 2004;78:1761-8.

8. Liu J, Cui F, Li S, et al. Nonintubated video-assisted thoracoscopic surgery under epidural anesthesia compared with conventional anesthetic option: a randomized control study. Surg Innov 2015;22:123-30.

9. Dong Q, Liang L, Li Y, et al. Anesthesia with nontracheal intubation in thoracic surgery. J Thorac Dis
2012;4:126-30.

10. Shao $W$, Wang $W$, Yin $W$, et al. Nonintubated thoracoscopic lobectomy plus lymph node dissection following segmentectomy for central type pulmonary masses. Chin J Cancer Res 2013;25:124-7.

11. Guo Z, Yin W, Wang W, et al. Spontaneous ventilation anaesthesia: total intravenous anaesthesia with local anaesthesia or thoracic epidural anaesthesia for thoracoscopic bullectomy. Eur J Cardiothorac Surg 2016;50:927-32.

12. Jiang L, Liu J, Shao W, et al. Non-intubated subxiphoid uniportal video-assisted thoracoscopic thymectomy using glasses-free 3D vision. J Thorac Dis 2016;8:E1602-4.

13. Li S, Liu J, He J, et al. Video-assisted transthoracic surgery resection of a tracheal mass and reconstruction of trachea under non-intubated anesthesia with spontaneous breathing. J Thorac Dis 2016;8:575-85.

14. Peng G, Cui F, Ang KL, et al. Non-intubated combined with video-assisted thoracoscopic in carinal reconstruction. J Thorac Dis 2016;8:586-93.

15. Huang J, Qiu Y, Chen L, et al. Nonintubated Spontaneous Respiration Anesthesia for Tracheal Glomus Tumor. Ann Thorac Surg 2017;104:e161-3.

16. Jiang L, Liu J, Gonzalez-Rivas D, et al. Thoracoscopic surgery for tracheal and carinal resection and reconstruction under spontaneous ventilation. J Thorac Cardiovasc Surg 2018;155:2746-54.

17. Morishita M, Watanabe H, Yan M, et al. Grade based on Common Terminology Criteria for Adverse Events Version 4.0. 2017. Available online: https://ctep.cancer. gov/protocoldevelopment/electronic_applications/docs/ CTCAE_4.03.xlsx
Cite this article as: Cui F, Xu K, Liang H, Liang W, Li J, Wang W, Liu H, Liu J, He J. Spontaneous ventilation versus mechanical ventilation during video-assisted thoracoscopic surgery for spontaneous pneumothorax: a study protocol for multicenter randomized controlled trial. J Thorac Dis 2020;12(4):1570-1581. doi: 10.21037/jtd.2020.02.13 